\title{
El mobbing como expresión de violencia física y psicológica en espacios universitarios: una aproximación desde una perspectiva sistemática
}

\begin{abstract}
Mobbing as an Expression of Physical and Psychological Violence in University Spaces: An Approach from a Systematic Perspective
\end{abstract}

Mobbing como expressão da violência física e psicológica nos espaços universitários: uma abordagem sob uma perspectiva sistemática

Brenda García López

Hospital General de Culiacán "Dr. Bernardo J. Gastelum”, México Hospital Regional ISSSTE “Dr. Manuel Cárdenas de la Vega”, México Universidad Autónoma de Sinaloa, México brenda7272@hotmail.com https://orcid.org/0000-0002-6381-9107

Alma Delia Herenas Tapia

Hospital General de Culiacán "Dr. Bernardo J. Gastelum", México Hospital Regional ISSSTE “Dr. Manuel Cárdenas de la Vega”, México Universidad Autónoma de Sinaloa, México almadeliaherenas@gmail.com https://orcid.org/0000-0002-0331-3577 Jesús Roberto Garay Núñez Universidad Autónoma de Sinaloa, México jrgarayn@hotmail.com https://orcid.org/0000-0002-0868-1344 


\section{Resumen}

La prevalencia del mobbing como expresión de violencia física y psicológica en las instituciones de educación superior de México y del mundo en los últimos años ha tenido un incremento exponencial. La interacción social entre los diferentes actores que conviven en los espacios universitarios pone de relieve las estructuras de poder que se generan en torno a dicho fenómeno. El objetivo de esta investigación fue analizar la literatura en español que se centra en el mobbing, en específico aquella realizada en el periodo de 2006 a 2019 y cuyo contexto es el espacio universitario. Se utilizaron métodos sistemáticos y explícitos para localizar, seleccionar y valorar críticamente las investigaciones relevantes, según las orientaciones derivadas de la Declaración Prisma. Las bases de datos que se consultaron fueron Redalyc, Scielo, Scopus y ebsco. A partir de lo anterior, se seleccionaron y analizaron ocho artículos para determinar el mobbing en instituciones de educación superior de México y de Iberoamérica. Entre las conclusiones destaca que el mobbing es un acto hostil que se puede manifestar en todos los campos de relación humana presentes en la universidad, y en las instituciones de educación superior mexicanas su presencia se ha multiplicado.

Palabras claves: espacio educativo, estrés, mobbing, salud mental.

\section{Abstract}

The prevalence of mobbing as an expression of physical and psychological violence in higher education institutions in Mexico and in the world in recent years has had an exponential increase. The social interaction between the different actors that coexist in the university spaces highlights the power structures that are generated around this phenomenon. The objective of this research was to analyze the literature in Spanish that focuses on mobbing, specifically that carried out in the period from 2006 to 2019 and whose context is the university space. Systematic and explicit methods were used to locate, select and critically appraise the relevant investigations, according to the guidelines derived from the Prism Declaration. The databases that were consulted were Redalyc, Scielo, Scopus and ebsco. Based on the above, eight articles were selected and analyzed to determine mobbing in higher education institutions in Mexico and Latin America. Among the conclusions, it stands out that mobbing is a hostile act that can manifest itself in all the fields of human relationship 
present in the university, and in the Mexican higher education institutions its presence has multiplied.

Keywords: educational space, stress, mobbing, mental health.

\section{Resumo}

A prevalência de assédio moral como expressão de violência física e psicológica em instituições de ensino superior no México e no mundo nos últimos anos teve um aumento exponencial. A interação social entre os diferentes atores que coexistem nos espaços universitários destaca as estruturas de poder geradas em torno desse fenômeno. O objetivo desta pesquisa foi analisar a literatura em espanhol que se concentra no assédio moral, especificamente aquela realizada no período de 2006 a 2019 e cujo contexto é o espaço universitário. Métodos sistemáticos e explícitos foram utilizados para localizar, selecionar e avaliar criticamente as investigações relevantes, de acordo com as diretrizes derivadas da Declaração de Prisma. As bases de dados consultadas foram Redalyc, Scielo, Scopus e ebsco. Com base no exposto, oito artigos foram selecionados e analisados para determinar o assédio moral em instituições de ensino superior no México e na América Latina. Entre as conclusões, destaca-se que o assédio moral é um ato hostil que pode se manifestar em todos os campos do relacionamento humano presentes na universidade, e nas instituições de ensino superior mexicanas sua presença se multiplicou.

Palavras-chave: espaço educacional, estresse, assédio moral, saúde mental. 


\section{Introducción}

El término mobbing proviene del verbo inglés to mob, que se traduce como 'regañar, atacar, maltratar, asediar', mientras que mob como sustantivo significa 'muchedumbre, jauría'. De esta manera, el término expone un fenómeno de grupo (Hirigoyen, 2001).

El mobbing es la comunicación hostil y desprovista de ética que es administrada de forma sistemática por uno o unos pocos individuos principalmente contra un único individuo, quien, a consecuencia de ello, es arrojado a una situación de soledad e indefensión prolongada. Se trata de acciones de hostigamiento frecuentes y persistentes (definición estadística: al menos una vez por semana) a lo largo de un extenso período (definición estadística: al menos durante seis meses) (Leymann, 1996).

En México, el estudio de la violencia en el nivel superior es muy reciente. En 2012, el Consejo Nacional de Ciencia y Tecnología (Conacyt) publicó una convocatoria para promover la investigación sobre la violencia dentro de las universidades y atender la urgencia de diagnosticar, analizar y prevenir esta problemática. El tema se ha vuelto necesario no solo porque "la violencia ha afectado al interior de estos espacios, sino también porque dentro del mismo se reproducen casi todas las formas de violencia que se viven en otros escenarios" (Carrillo, 2015).

Muchas instituciones de educación superior se han dado a la tarea de generar estrategias para prevenir, disminuir y erradicar este problema; sin embargo, existen pocos trabajos que ofrezcan información sobre dicho fenómeno y, por extensión, que fomenten mejores formas de convivencia capaces de reducir al mínimo la violencia en sus múltiples manifestaciones (Montesinos y Carrillo, 2011).

Múltiples manifestaciones porque, efectivamente, en el contexto de pregrado y posgrado, en el ámbito académico/profesional y laboral, la violencia que ejercen, reciben y mantienen los diferentes actores universitarios (alumnos, docentes, autoridades, administrativos y trabajadores) es polifacética: psicológica, social, física, sexual, virtual, patrimonial y económica (Tlalolin, 2017). Las interacciones disfuncionales entre ellos (multidimensionalidad de la violencia) se manifiestan en conductas de acción u omisión que tienen la intención de producir daño biopsicosocial (físico, psicológico y social) (Tlalolin, 2017). 
Revista Iberoamericana

de las Ciencias Sociales y

Humanísticas

ISSN: $2395-7972$

Las manifestaciones del mobbing varían con respecto a la disposición de las condiciones laborales. Asimismo, las conductas más comunes abarcan desde gritos, discriminación e insultos directos hasta señales y miradas obscenas, y en los casos de mayor intensidad, agresiones físicas graves; aún más, en casos extremos, se puede presentar en forma de homicidio (Bowling y Beehr, 2006).

Respecto al acoso dentro del personal universitario, se entiende que toda institución educativa representa un espacio en el que las interacciones influyen en el desempeño laboral y en el bienestar de los involucrados (Moreno, Sepúlveda y Restrepo, 2012).

El objetivo de esta revisión sistemática es analizar el fenómeno del mobbing como expresión de violencia física y psicológica en espacios universitarios en el periodo de 2006 a 2019.

\section{Materiales y métodos}

Para el análisis y revisión de los artículos se utilizaron la palabra y la cadena de términos mobbing y violencia física y psicológica en espacios universitarios. Se utilizaron métodos sistemáticos y explícitos para localizar, seleccionar y valorar críticamente las investigaciones relevantes, según las orientaciones derivadas de la Declaración Prisma (Hutton, Catalá y Moher, 2016). Las bases de datos consultadas fueron Redalyc, Scielo, Scopus y ebsco.

La búsqueda se limitó a artículos publicados en el rango de 2006 a 2019 disponibles en español para su análisis completo.

\section{Criterios de inclusión}

Se consideraron tres criterios de inclusión previamente definidos: 1) los participantes, 2) el concepto y 3) el contexto. En cuanto al tipo de participantes, se consideraron todos los estudios que se enfocan en los docentes, estudiantes y personal administrativo de ambos géneros. En cuanto el concepto, se consideraron los estudios con descriptores como mobbing, violencia física y violencia psicológica; y en cuanto al contexto, se consideraron estudios realizados en cualquier espacio que se considere universitario o instituciones de educación superior. Así, se seleccionaron ocho artículos que cumplieron con los criterios de inclusión descritos anteriormente. 


\section{Resultados}

En la tabla 1 podemos encontrar a los autores de los ocho artículos que se seleccionaron para analizar el fenómeno del mobbing en instituciones de educación superior. En cuanto al objetivo principal, se determinó que $37.5 \%$ está dirigido a determinar el acoso laboral, $12.5 \%$ el clima laboral, $12.5 \%$ a identificar la prevalencia de mobbing, $12.5 \%$ a identificar su impacto en el autoestima, $12.5 \%$ a identificar los tipos de violencia y $12.5 \%$ a correlacionar el mobbing con la satisfacción laboral. La mitad de los artículos se abordaron desde la perspectiva cuantitativa; en $37.5 \%$ se realizó una revisión sistemática, y en $12.5 \%$ se recurrió a un diseño metodológico mixto. En cuanto a los aspectos éticos de la investigación, solo $37.5 \%$ especifica claramente este apartado; el restante $62.5 \%$ no lo menciona. 
Revista Iberoamericana

de las Ciencias Sociales y

Humanísticas

Tabla 1. Principales categorías de los artículos analizados.

\begin{tabular}{|c|c|c|c|}
\hline Autor(es) y año & Objetivo & Enfoque/Diseño & $\begin{array}{l}\text { Procedimientos } \\
\text { éticos }\end{array}$ \\
\hline Gómez (2017) & $\begin{array}{l}\text { Abordar el acoso } \\
\text { laboral en la } \\
\text { universidad. }\end{array}$ & Análisis comparado & No se mencionan. \\
\hline $\begin{array}{l}\text { López, Picón y } \\
\text { Vázquez (2008) }\end{array}$ & $\begin{array}{l}\text { Caracterizar los } \\
\text { procesos de acoso } \\
\text { psicológico } \\
\text { entre el personal } \\
\text { de administración } \\
\text { y servicios de la } \\
\text { universidad } \\
\text { pública de Galicia. }\end{array}$ & Cuantitativo & No se mencionan. \\
\hline $\begin{array}{l}\text { Justicia, Benítez y } \\
\text { Fernández de Haro } \\
\text { (2006) }\end{array}$ & $\begin{array}{l}\text { Caracterizar el } \\
\text { fenómeno del } \\
\text { acoso psicológico } \\
\text { en el contexto } \\
\text { específico de la } \\
\text { universidad. }\end{array}$ & Cuantitativo & $\begin{array}{l}\text { Para tal fin, y para } \\
\text { salvaguardar la } \\
\text { identidad del } \\
\text { participante, se } \\
\text { asoció de forma } \\
\text { aleatoria un código } \\
\text { de acceso a cada } \\
\text { una de las } \\
\text { direcciones de } \\
\text { correo electrónico. } \\
\text { El control de acceso } \\
\text { al cuestionario } \\
\text { estaba doblemente } \\
\text { justificado. } \\
\text { Primero, para, } \\
\text { como ya se ha } \\
\text { mencionado, } \\
\text { preservar la } \\
\text { identidad y el } \\
\text { anonimato de } \\
\text { cuantos pudieran } \\
\text { participar con sus } \\
\text { respuestas en el } \\
\text { estudio. Segundo, } \\
\text { para garantizar que } \\
\text { solo respondieran } \\
\text { trabajadores de las } \\
\text { universidades } \\
\text { participantes y que } \\
\text { estos lo hicieran } \\
\text { una sola vez. }\end{array}$ \\
\hline
\end{tabular}


Revista Iberoamericana

de las Ciencias Sociales y Humanísticas

ISSN: $2395-7972$

\begin{tabular}{|c|c|c|c|}
\hline $\begin{array}{l}\text { Ibarra, Escalante, } \\
\text { y Mendizábal } \\
(2015)\end{array}$ & $\begin{array}{l}\text { Aportar una } \\
\text { propuesta para } \\
\text { lograr un cambio } \\
\text { en el clima laboral } \\
\text { de la comunidad } \\
\text { universitaria de la } \\
\text { Universidad } \\
\text { Autónoma del } \\
\text { Estado de Morelos } \\
\text { (UAEM), } \\
\text { extendiendo su } \\
\text { impacto a otras } \\
\text { instituciones del } \\
\text { estado y del país. }\end{array}$ & Análisis sistemático & No se mencionan. \\
\hline $\begin{array}{l}\text { Montesinos y } \\
\text { Carrillo (2011) }\end{array}$ & $\begin{array}{l}\text { Realizar un } \\
\text { levantamiento de } \\
\text { información que } \\
\text { recoja la voz de } \\
\text { todos los actores } \\
\text { de la comunidad } \\
\text { universitaria: } \\
\text { alumnos, alumnas, } \\
\text { académicos, } \\
\text { académicas, } \\
\text { funcionarios, } \\
\text { funcionarias, } \\
\text { trabajadores y } \\
\text { trabajadoras, sobre } \\
\text { las diferentes } \\
\text { formas de } \\
\text { violencia que } \\
\text { experimentan en su } \\
\text { interacción en el } \\
\text { espacio } \\
\text { universitario. }\end{array}$ & $\begin{array}{l}\text { Cualitativo y } \\
\text { cuantitativo }\end{array}$ & $\begin{array}{l}\text { Se cambió el } \\
\text { nombre de los } \\
\text { participantes para } \\
\text { proteger su } \\
\text { identidad. }\end{array}$ \\
\hline $\begin{array}{l}\text { Seijo y Nava } \\
(2016)\end{array}$ & $\begin{array}{l}\text { Analizar las } \\
\text { consecuencias del } \\
\text { psicoterror hacia el } \\
\text { ser humano con la } \\
\text { intención de } \\
\text { desestabilizarlo y } \\
\text { destruir su } \\
\text { autoestima } \\
\text { afectando } \\
\text { automáticamente } \\
\text { la salud mental. }\end{array}$ & Análisis sistemático & No se mencionan. \\
\hline Tlalolin (2017) & $\begin{array}{l}\text { Describir los tipos } \\
\text { de violencias que }\end{array}$ & Cuantitativo & $\begin{array}{l}\text { Se asegura la } \\
\text { confidencialidad y }\end{array}$ \\
\hline
\end{tabular}


Revista Iberoamericana

de las Ciencias Sociales y

Humanísticas

ISSN: $2395-7972$

\begin{tabular}{|c|c|c|c|}
\hline & $\begin{array}{l}\text { viven } \\
\text { cotidianamente los } \\
\text { estudiantes del } \\
\text { área de ciencias } \\
\text { sociales y } \\
\text { humanidades de la } \\
\text { Benemérita } \\
\text { Universidad } \\
\text { Autónoma de } \\
\text { Puebla (BUAP). }\end{array}$ & & $\begin{array}{l}\text { anonimato de los } \\
\text { participantes. }\end{array}$ \\
\hline $\begin{array}{l}\text { Muñoz y López } \\
\text { (2018) }\end{array}$ & $\begin{array}{l}\text { Determinar la } \\
\text { existencia de } \\
\text { dependencia } \\
\text { estadística entre las } \\
\text { variables Mobbing } \\
\text { y Satisfacción } \\
\text { Laboral en } \\
\text { docentes } \\
\text { universitarios de } \\
\text { cuatro } \\
\text { instituciones } \\
\text { del centro de } \\
\text { México. }\end{array}$ & Cuantitativo & No lo mencionan. \\
\hline
\end{tabular}

Fuente: Elaboración propia

En la tabla 2 encontramos que $62.5 \%$ de los artículos se realizaron en México, $25 \%$ en España y $12.5 \%$ en Colombia. El tipo de población que se encontró es muy diversa. La mitad, 50\%, realizó encuestas a estudiantes, docentes y personal administrativo de instituciones de educación superior. Por otro lado, $37.5 \%$ analizó el contenido de 56 artículos y se elaboró un ensayo. La prevalencia e incidencia del mobbing se puede apreciar principalmente en la variable de violencia psicológica en la modalidad de acoso laboral. 
Revista Iberoamericana

de las Ciencias Sociales y

Humanísticas

ISSN: $2395-7972$

Tabla 2. País de origen, tamaño de la muestra y prevalencia de mobbing

\begin{tabular}{|c|c|c|c|}
\hline Autor(es) y año & País & $\begin{array}{l}\text { Tamaño de la } \\
\text { muestra }\end{array}$ & Prevalencia/Incidencia \\
\hline Gómez (2017) & México & 50 artículos & $\begin{array}{l}\text { El abuso de autoridad } \\
\text { con un porcentaje de } \\
14.8 \% \text {, el acoso laboral } \\
\text { con } 13.6 \% \text { y la } \\
\text { exclusión con } 11.4 \% \text {. }\end{array}$ \\
\hline $\begin{array}{l}\text { López et al. } \\
\text { (2008) }\end{array}$ & España & 321 personas & $\begin{array}{l}8.7 \% \\
\text { manifiesta ser víctimas } \\
\text { de mobbing. }\end{array}$ \\
\hline $\begin{array}{l}\text { Justicia et al. } \\
\text { (2006) }\end{array}$ & España & 548 personas & $\begin{array}{l}9.31 \% \text { de los } \\
\text { empleados } \\
\text { universitarios se } \\
\text { considera víctima del } \\
\text { acoso. }\end{array}$ \\
\hline Ibarra et al. (2015) & México & Seis artículos & No aplica. \\
\hline $\begin{array}{l}\text { Montesinos y } \\
\text { Carrillo (2011) }\end{array}$ & México & $\begin{array}{l}\text { En la universidad: } \\
2216 \text { cuestionarios } \\
\text { a estudiantes, } 26 \\
\text { historias de vida } \\
\text { de estudiantes, } \\
\text { cuatro historias de } \\
\text { vida de profesores, } \\
\text { tres historias de } \\
\text { vida de } \\
\text { trabajadores, dos } \\
\text { historias laborales } \\
\text { de trabajadoras, } \\
\text { cuatro historias de } \\
\text { vida de } \\
\text { funcionarios y una } \\
\text { historia laboral de } \\
\text { un profesor; } \\
\text { cuatro grupos } \\
\text { focales } \\
\text { compuestos de } \\
\text { estudiantes, } 15 \\
\text { entrevistas a } \\
\text { profesores, } 35 \\
\text { entrevistas a } \\
\text { estudiantes. }\end{array}$ & Discursiva. No aplica. \\
\hline $\begin{array}{l}\text { Seijo y Nava } \\
(2016)\end{array}$ & Colombia & Ensayo & No aplica. \\
\hline Tlalolin (2017) & México & 150 estudiantes & $\begin{array}{l}\text { Las manifestaciones de } \\
\text { violencia psicológica y } \\
\text { social fueron las que }\end{array}$ \\
\hline
\end{tabular}




\begin{tabular}{|l|l|l|l|}
\hline & & & $\begin{array}{l}\text { tuvieron mayor } \\
\text { prevalencia. Es } \\
\text { importante señalar que } \\
\text { estas violencias tienen } \\
\text { un carácter simbólico } \\
\text { que, al no dejar marcas } \\
\text { visibles en las víctimas, } \\
\text { dificultan su } \\
\text { identificación, lo que } \\
\text { muchas veces provoca } \\
\text { que no se tengan } \\
\text { pruebas fehacientes } \\
\text { para denunciarlas. }\end{array}$ \\
& & & $\begin{array}{l}\text { Satisfacción laboral y } \\
\text { mobbing }\end{array}$ \\
\hline $\begin{array}{l}\text { Muñoz y López } \\
\text { (2018) }\end{array}$ & México & $\begin{array}{l}\text { 248 docentes de } \\
\text { cuatro } \\
\text { instituciones de } \\
\text { educación } \\
\text { superior. }\end{array}$ & \\
\hline & & & \\
\hline
\end{tabular}

Fuente: Elaboración propia

En la tabla 3 se describen los instrumentos que se utilizaron para recabar la información sobre el mobbing. Como se puede apreciar, $25 \%$ utilizó el LIPT- 60 de Leymann; $25 \%$ utilizó un cuestionario sobre acoso psicológico; $12.5 \%$ utilizó la saturación teórica para enfoque cualitativo; $12.5 \%$ se decantó por la revisión teórica, y $12.5 \%$ diseñó un instrumento ad hoc para su implementación. Con respecto a la validación de los instrumentos, se encontró que $75 \%$ de los estudios no hace mención del mecanismo de validación de la prueba que utilizó, $25 \%$ sí lo menciona y $12.5 \%$ lo hace a través de la saturación teórica de su prueba piloto. 
Revista Iberoamericana

de las Ciencias Sociales y Humanísticas

Tabla 3. Instrumentos utilizados y validación de los estudios

\begin{tabular}{|c|c|c|}
\hline Autor(es) y año & Instrumento(s) & Validación \\
\hline Gómez (2017) & LIPT- 60 de Leymann. & No se menciona. \\
\hline López et al. (2008) & $\begin{array}{l}\text { Cuestionario sobre } \\
\text { acoso psicológico. La } \\
\text { primera parte del } \\
\text { cuestionario consta de } \\
13 \text { preguntas relativas a la } \\
\text { universidad y aspectos } \\
\text { sociodemográficos. La } \\
\text { siguiente parte consta de } \\
\text { cinco bloques: } 1 \text { ) } \\
\text { identificación de las } \\
\text { víctimas, 2) } \\
\text { identificación de las } \\
\text { conductas sufridas, 3) } \\
\text { atribuciones causales, 4) } \\
\text { consecuencias sobre la } \\
\text { salud de la víctima y 5) } \\
\text { respuesta de la víctima } \\
\text { ante los ataques. } \\
\\
\text { En el apartado de dedicado } \\
\text { a las consecuencias sobre } \\
\text { la salud, los } 42 \text { ítems que } \\
\text { lo componen se han } \\
\text { medido mediante la } \\
\text { adaptación al español del } \\
\text { cuestionario de medida de } \\
\text { mobbing de Lazzari (2001, } \\
\text { citado en López et al., } \\
\text { 2008). } \\
\text { Mientras que en el de } \\
\text { respuesta de la víctima, los } \\
11 \text { ítems que lo componen } \\
\text { se han medido a través de } \\
\text { una adaptación de la escala } \\
\text { propuesta por Piñuel y } \\
\text { Oñate (2003, citados en } \\
\text { López et al., 2008). }\end{array}$ & $\begin{array}{l}\text { No se han analizado las } \\
\text { propiedades psicométricas } \\
\text { de las escalas debido a que } \\
\text { no han sido elaboradas con } \\
\text { una intención diagnóstica, } \\
\text { sino más bien exploratoria, } \\
\text { con el objeto de conocer } \\
\text { mejor las características } \\
\text { del mobbing en el contexto } \\
\text { universitario. }\end{array}$ \\
\hline Justicia et al. (2006) & $\begin{array}{l}\text { Cuestionario para el } \\
\text { Estudio del Acoso Laboral } \\
\text { en Contextos } \\
\text { Universitarios (Justicia, } \\
\text { Fernández, Benítez, } \\
\text { Villena, García y Caurcel, }\end{array}$ & $\begin{array}{l}\text { La primera parte recoge } \\
\text { datos generales de los } \\
\text { encuestados. El } \\
\text { cuestionario presenta una } \\
\text { segunda parte con } 36 \\
\text { ítems (alfa de Cronbach = }\end{array}$ \\
\hline
\end{tabular}




\begin{tabular}{|c|c|c|}
\hline & $\begin{array}{l}\text { 2002, citado en Justicia et } \\
\text { al., 2006) construido ad } \\
\text { hoc para tal fin. }\end{array}$ & $\begin{array}{l}\text { 0.8605) distribuidos en } \\
\text { cuatro bloques: } 1 \text { ) } \\
\text { caracterización de } \\
\text { fenómeno (12 ítems); } 2 \text { ) } \\
\text { caracterización del acoso } \\
\text { según las víctimas (19 } \\
\text { ítems); } 3 \text { ) caracterización } \\
\text { del acoso según los } \\
\text { testigos ( } 2 \text { ítems), y 4) } \\
\text { caracterización del } \\
\text { acosador (3 ítems). La } \\
\text { validez del instrumento } \\
\text { fue testada a través del } \\
\text { juicio de seis expertos } \\
\text { pertenecientes al } \\
\text { Departamento de } \\
\text { Psicología Evolutiva y de } \\
\text { la Educación y dos } \\
\text { expertos en Métodos y } \\
\text { Diseños de Investigación } \\
\text { de la Universidad de } \\
\text { Granada. }\end{array}$ \\
\hline Ibarra et al. (2015) & LIPT- 60 de Leymann. & No lo menciona. \\
\hline $\begin{array}{l}\text { Montesinos y Carrillo } \\
\text { (2011) }\end{array}$ & $\begin{array}{l}\text { Cuestionarios, entrevistas } \\
\text { y grupos } \\
\text { focales. }\end{array}$ & No lo mencionan. \\
\hline Seijo y Nava (2016) & Análisis y reflexión. & No aplica. \\
\hline Tlalolin (2017) & $\begin{array}{l}\text { Se diseñó un cuestionario } \\
\text { ad hoc compuesto de } \\
\text { cuatro baterías que } \\
\text { evaluaron las interacciones } \\
\text { alumno-alumno, profesor- } \\
\text { alumno, administrativo- } \\
\text { alumno y trabajador- } \\
\text { alumno. }\end{array}$ & No lo mencionan. \\
\hline Muñoz y López (2018) & $\begin{array}{l}\text { El primer cuestionario } \\
\text { correspondió a mobbing y } \\
\text { fue desarrollado a partir } \\
\text { del Cuestionario } \\
\text { Interpersonal sobre } \\
\text { Psicoterror, Ninguneo, } \\
\text { Estigmatización y Rechazo } \\
\text { en Organizaciones } \\
\text { Sociales (Cisneros) de } \\
\text { Iñaki Piñuel. El segundo } \\
\text { instrumento, que } \\
\text { correspondió a satisfacción } \\
\text { laboral, tomó como base la }\end{array}$ & $\begin{array}{l}\text { Mediante el método de } \\
\text { consistencia interna } \\
\text { basado en el Alfa de } \\
\text { Cronbach, o dimensión } \\
\text { teórica. Los valores } \\
\text { obtenidos fueron de } 0.89 \\
\text { para el instrumento de } \\
\text { recolección de datos } \\
\text { correspondiente a } \\
\text { mobbing, y de } 0.91 \text { para el } \\
\text { de satisfacción laboral. }\end{array}$ \\
\hline
\end{tabular}




\begin{tabular}{|l|l|}
\hline Escala Multidimensional & \\
de Satisfacción Laboral & \\
Docente (EMSLD) & (Barraza y Ortega, 2009, \\
citados en Muñoz y López, & \\
2018) y la Escala de & \\
Satisfacción Laboral- & Versión para Profesores \\
(ESL-VP) (Anaya y & \\
Suárez, 2007, citados en & \\
Muñoz y López, 2018). & \\
\hline
\end{tabular}

Fuente: Elaboración propia

En la tabla 4 encontramos el tipo de influencia que puede favorecer el mobbing: los factores culturales con $12.5 \%$; el personal/profesional con $25 \%$; la lucha de poder con $12.5 \%$; la esfera laboral y profesional con $12.5 \%$; influencias sociales con $12.5 \%$; variables relacionadas a la autoestima con $12.5 \%$, y algún tipo de violencia con $12.5 \%$. En cuanto a los factores de riesgo percibidos, se encontró que el género está relacionado en $75 \%$; el clima laboral en $12.5 \%$, y el acoso laboral con $12.5 \%$. En lo que respecto al principal tipo de acoso percibido, se determinó que $50 \%$ está relacionado con la salud psicológica; algún tipo de violencia con $25 \%$; acoso laboral con $12.5 \%$, y se sienten explotadas con $12.5 \%$. 
Revista Iberoamericana

de las Ciencias Sociales y

Humanísticas

ISSN: $2395-7972$

Tabla 4. Influencias, factores de riesgo y principal tipo de acoso percibido

\begin{tabular}{|c|c|c|c|}
\hline Autor(es) y año & Influencias & Factores de riesgo & $\begin{array}{l}\text { Principal acoso } \\
\text { percibido }\end{array}$ \\
\hline Gómez (2017) & Culturales & Acoso laboral & $\begin{array}{l}\text { Se sienten } \\
\text { explotadas en su } \\
\text { trabajo; } 7.3 \% \\
\text { refiere que se les } \\
\text { da trabajo en un } \\
\text { nivel inferior a su } \\
\text { nivel de } \\
\text { competencia, y } \\
6.7 \% \text { informa que } \\
\text { se les oculta } \\
\text { información } \\
\text { necesaria para } \\
\text { realizar su trabajo. }\end{array}$ \\
\hline López et al. (2008) & $\begin{array}{l}\text { Tanto la esfera } \\
\text { personal como con } \\
\text { la profesional. }\end{array}$ & Clima laboral & $\begin{array}{l}\text { Alteración a la } \\
\text { salud psicológica y } \\
\text { emocional. }\end{array}$ \\
\hline $\begin{array}{l}\text { Justicia et al. } \\
\text { (2006) }\end{array}$ & $\begin{array}{l}\text { Luchas de poder, la } \\
\text { envidia, la } \\
\text { prepotencia del } \\
\text { acosador y la } \\
\text { competitividad. }\end{array}$ & $\begin{array}{l}\text { Género y colectivo } \\
\text { profesional }\end{array}$ & $\begin{array}{l}\text { La salud física, la } \\
\text { salud emocional, la } \\
\text { actividad } \\
\text { profesional y las } \\
\text { relaciones sociales. }\end{array}$ \\
\hline Ibarra et al. (2015) & $\begin{array}{l}\text { Esfera laboral y } \\
\text { esfera institucional. }\end{array}$ & Género & Acoso laboral \\
\hline $\begin{array}{l}\text { Montesinos y } \\
\text { Carrillo (2011) }\end{array}$ & Sociales & Género & $\begin{array}{l}\text { Violencia entre } \\
\text { hombres y mujeres. }\end{array}$ \\
\hline $\begin{array}{l}\text { Seijo y Nava } \\
\text { (2016) }\end{array}$ & Autoestima & Género & $\begin{array}{l}\text { Violencia y salud } \\
\text { mental. }\end{array}$ \\
\hline Tlalolin (2017) & $\begin{array}{l}\text { Violencia } \\
\text { psicológica, física, } \\
\text { sexual, patrimonial, } \\
\text { económica y la } \\
\text { ciberviolencia. }\end{array}$ & Género & $\begin{array}{l}\text { Los alumnos han } \\
\text { sido víctimas de } \\
\text { violencia social } \\
(31.5 \%) \text {, } \\
\text { psicológica }(30 \%) \text {, } \\
\text { sexual }(16.8 \%) \text {, } \\
\text { física }(8.7 \%) \text {, } \\
\text { virtual }(8.9 \%) \text { y } \\
\text { patrimonial }(3.9 \%) \\
\text { por parte de otros } \\
\text { estudiantes. }\end{array}$ \\
\hline $\begin{array}{l}\text { Muñoz y López } \\
\text { (2018) }\end{array}$ & $\begin{array}{l}\text { Personal, } \\
\text { organizacional y } \\
\text { sobrecarga de } \\
\text { trabajo. }\end{array}$ & Género & $\begin{array}{l}\text { Factores } \\
\text { psicológicos, } \\
\text { subjetivos, } \\
\text { simbólicos y éticos }\end{array}$ \\
\hline
\end{tabular}

Fuente: Elaboración propia 
Revista Iberoamericana

de las Ciencias Sociales y

Humanísticas

ISSN: $2395-7972$

En la tabla 5 podemos encontrar los resultados principales de estas investigaciones. Allí se pone de manifiesto que la prevalencia sobre mobbing y la violencia física y psicológica son fenómenos que se están presentando e incrementando en los diferentes espacios de las instituciones de educación superior. También en la tabla 5 aparecen las conclusiones de los estudios, así como algunas propuestas de mejora continua en el clima laboral que impera en nuestras instituciones educativas.

Tabla 5. Principales resultados y conclusiones de los estudios realizados

\begin{tabular}{|c|c|c|}
\hline Autor(es) y año & Resultados & Conclusiones \\
\hline Gómez (2017) & $\begin{array}{l}\text { Se destaca que al menos } \\
79.5 \% \text { de los hombres y } \\
83.6 \% \text { de las mujeres } \\
\text { considera haber sufrido al } \\
\text { menos una estrategia de } \\
\text { acoso. }\end{array}$ & $\begin{array}{l}\text { La existencia de un } \\
\text { ambiente hostil de trabajo } \\
\text { genera diferentes tipos de } \\
\text { conflictos interpersonales } \\
\text { y relaciones negativas } \\
\text { entre los compañeros de } \\
\text { trabajo, que afectan el } \\
\text { bienestar y la } \\
\text { salud de los trabajadores. }\end{array}$ \\
\hline López et al. (2008) & $\begin{array}{l}\text { Las manifestaciones de } \\
\text { acoso psicológico más } \\
\text { frecuentes son: no } \\
\text { transmitir informaciones } \\
\text { útiles ( } 66 \% \text { de los } \\
\text { acosados señalan padecer } \\
\text { esta conducta); no hablar } \\
\text { ni reunirse con el acosado } \\
\text { (53.6\% ); lesionar la } \\
\text { autoestima (53.6 \%); } \\
\text { controlar en exceso el } \\
\text { trabajo de la víctima } \\
\text { (46.4 \%); infravalorar su } \\
\text { esfuerzo profesional } \\
\text { (46.4 \%); criticar } \\
\text { exageradamente su trabajo } \\
\text { (46.4 \%), evaluándolo } \\
\text { negativamente (42.9 \%); } \\
\text { desacreditar a la víctima } \\
\text { delante de otros (42.9 \%), } \\
\text { amplificando nimiedades y } \\
\text { acusándola de errores y } \\
\text { fallos difusos (42.9\%). }\end{array}$ & $\begin{array}{l}\text { La estrategia elegida } \\
\text { mayoritariamente por las } \\
\text { víctimas es no hacer nada } \\
\text { (pasiva), hablar con el } \\
\text { acosador (activa y } \\
\text { asertiva) y ponerlo en } \\
\text { conocimiento de la } \\
\text { organización o los órganos } \\
\text { de representación sindical } \\
\text { (denuncia inicial). }\end{array}$ \\
\hline Justicia et al. (2006) & $\begin{array}{l}\text { Los resultados obtenidos } \\
\text { de la muestra de } \\
\text { participantes indican que }\end{array}$ & $\begin{array}{l}\text { El porcentaje de víctimas } \\
\text { encontrado es ligeramente } \\
\text { inferior a los señalados en }\end{array}$ \\
\hline
\end{tabular}




\begin{tabular}{|c|c|c|}
\hline & $\begin{array}{l}22.3 \% \text { de ellos ha sufrido } \\
\text { acoso por lo menos una } \\
\text { vez durante su experiencia } \\
\text { laboral. Sin embargo, si } \\
\text { introducimos criterios de } \\
\text { valoración más } \\
\text { restrictivos, tales como la } \\
\text { frecuencia y la duración, el } \\
\text { porcentaje desciende hasta } \\
9.31 \% \text { de los } \\
\text { participantes. }\end{array}$ & $\begin{array}{l}\text { otros estudios que sitúan la } \\
\text { incidencia en el sector } \\
\text { educativo entre } 12 \% \text { y } \\
17 \% \text {. }\end{array}$ \\
\hline Ibarra et al. (2015) & $\begin{array}{l}\text { Las condiciones y } \\
\text { mutaciones que ha } \\
\text { experimentado el mundo } \\
\text { laboral en los últimos años } \\
\text { han generado ambientes } \\
\text { agresivos y de alta presión } \\
\text { para los trabajadores, con } \\
\text { las consecuencias que ello } \\
\text { deriva en lo personal y en } \\
\text { lo institucional. }\end{array}$ & $\begin{array}{l}\text { La legislación en materia } \\
\text { de acoso laboral establece } \\
\text { la base jurídica general en } \\
\text { cada país para atender a las } \\
\text { víctimas en los tribunales } \\
\text { y dar solución a los } \\
\text { conflictos que el acoso } \\
\text { laboral acarrea en los } \\
\text { centros de trabajo. }\end{array}$ \\
\hline $\begin{array}{l}\text { Montesinos y Carrillo } \\
\text { (2011) }\end{array}$ & $\begin{array}{l}\text { Formas de violencia de } \\
\text { género que se viven en la } \\
\text { universidad, } \\
\text { evidentemente dentro de la } \\
\text { violencia que se desata en } \\
\text { contra de las mujeres, sin } \\
\text { importar la relación de } \\
\text { poder entre uno y otro } \\
\text { actor, como es el caso de } \\
\text { la relación alumno- } \\
\text { trabajador y viceversa. }\end{array}$ & $\begin{array}{l}\text { Lo que enfrentamos en } \\
\text { ellas son prácticas cifras } \\
\text { en el abuso del poder, la } \\
\text { mediocridad académica y } \\
\text { burocrática, lo mismo que } \\
\text { una vida social carente de } \\
\text { compromiso y solidaridad } \\
\text { con los otros. }\end{array}$ \\
\hline Seijo y Nava (2016) & $\begin{array}{l}\text { Las víctimas de acoso van } \\
\text { más allá al afirmar que el } \\
\text { acto violento constituye un } \\
\text { atentado contra integridad } \\
\text { moral y ética de la } \\
\text { persona, en virtud de que } \\
\text { la actuación del acosador } \\
\text { va dirigida de modo } \\
\text { directo para someter y } \\
\text { manipular a la víctima, } \\
\text { con la intención de echarlo } \\
\text { del territorio. Aunque } \\
\text { algunas veces el psicópata } \\
\text { no consiga sacarlo de la } \\
\text { empresa, por lo menos le } \\
\text { queda la satisfacción de }\end{array}$ & $\begin{array}{l}\text { En el mundo laboral se } \\
\text { vive cada vez más el } \\
\text { incremento con respecto a } \\
\text { los daños psicológicos a la } \\
\text { salud mental ocasionados } \\
\text { por el fenómeno del } \\
\text { psicoterror, que poco a } \\
\text { poco destruye el } \\
\text { autoestima, el sentido de } \\
\text { pertenencia, la dignidad, } \\
\text { integridad y moral de } \\
\text { quien lo padece. }\end{array}$ \\
\hline
\end{tabular}




\begin{tabular}{|c|c|c|}
\hline & $\begin{array}{l}\text { hacerle la vida imposible, } \\
\text { con la mala impresión de } \\
\text { hacer pasar al acosado } \\
\text { como un trabajador } \\
\text { incompetente, } \\
\text { improductivo y } \\
\text { problemático. }\end{array}$ & \\
\hline Tlalolin (2017) & $\begin{array}{l}\text { Los resultados indican que } \\
\text { la violencia psicológica } \\
\text { ocupó el primer lugar de } \\
\text { incidencia al representar } \\
40.4 \% \text { del porcentaje } \\
\text { total. En segundo lugar se } \\
\text { encontró la violencia } \\
\text { social, la cual simbolizó } \\
35.5 \% \text { de las conductas } \\
\text { vividas por los } \\
\text { universitarios. La } \\
\text { violencia sexual se sitúa } \\
\text { en el tercer lugar con } \\
14.1 \% \text {. Los estudiantes } \\
\text { manifestaron haber sido } \\
\text { víctimas de comentarios } \\
\text { sexistas, bromas de } \\
\text { carácter sexual y miradas } \\
\text { lascivas. }\end{array}$ & $\begin{array}{l}\text { 1) Los estudiantes } \\
\text { universitarios son blanco } \\
\text { de diferentes tipos de } \\
\text { violencia, las cuales son } \\
\text { ejercidas por diversos } \\
\text { actores universitarios. } \\
\text { 2) Las manifestaciones de } \\
\text { violencia varían según qué } \\
\text { relación se evalúe. } \\
\text { 3) Es más probable que un } \\
\text { universitario identifique } \\
\text { más conductas violentas de } \\
\text { los actores con los que } \\
\text { convive con mayor } \\
\text { frecuencia, como en el } \\
\text { caso de los profesores o } \\
\text { alumnos que interactúan } \\
\text { constantemente. }\end{array}$ \\
\hline Muñoz y López (2018) & $\begin{array}{l}\text { La cantidad de docentes } \\
\text { con un nivel de } \\
\text { satisfacción laboral alto y } \\
\text { riesgo de mobbing muy } \\
\text { alto a medio es cero, y la } \\
\text { frecuencia de aquellos } \\
\text { docentes con un nivel de } \\
\text { satisfacción laboral bajo y } \\
\text { un riesgo bajo o sin riesgo } \\
\text { de mobbing es de solo de } \\
13 \text {. }\end{array}$ & $\begin{array}{l}\text { Los hallazgos permiten } \\
\text { observar la existencia de } \\
\text { una dependencia entre la } \\
\text { satisfacción laboral y el } \\
\text { mobbing, como se } \\
\text { esperaba, por lo que } \\
\text { generar estrategias para } \\
\text { aumentar la primera y } \\
\text { disminuir el segundo } \\
\text { resulta necesario para crear } \\
\text { un lugar más agradable } \\
\text { para trabajar, el cual } \\
\text { favorezca el desarrollo de } \\
\text { las personas y con ello de } \\
\text { las instituciones de } \\
\text { educación superior. }\end{array}$ \\
\hline
\end{tabular}

Fuente: Elaboración propia 


\section{Discusión}

La investigación de Rojas, García y Hernández (2019) reveló que la cantidad de estudios realizados sobre el tema del acoso laboral en trabajadores universitarios aún sigue siendo limitada, considerando el amplio rango de años que se utilizó para la realización del presente trabajo; aun así, se distingue un alza en la producción científica de este tema durante los últimos cinco años. En lo que se refiere a la distribución geográfica, se infiere una heterogeneidad con lo que respecta al país de realización (Rojas et al., 2019).

En nuestro análisis encontramos que las investigaciones sobre mobbing, violencia física y psicológica en espacios universitarios han tenido un notable incremento en el interés de profundizar sobre esta problemática en las instituciones de educación superior. Como ya se mencionó líneas arriba, $62.5 \%$ de los artículos revisados son de México, $25 \%$ de España y $12.5 \%$ de Colombia.

En México, el fenómeno del mobbing es poco conocido, y no se comprende en su totalidad el gran daño que ocasiona a la salud psicológica. Este tipo de discriminación afecta no solo a las personas que laboral en espacios universitarios, sino también puede atentar contra la dignidad de los estudiantes, que generalmente son la cadena más débil del eslabón.

La escuela todavía es vista como una de las pocas vías concretas de cambio y de movilidad social disponibles para una gran parte de la población. La idea de que la escuela es un sitio que debe ofrecer protección, y de que también es un lugar que tiene que ser preservado por la sociedad, ya no corresponde a la realidad de la mayoría de los establecimientos escolares. Por tal razón, las escuelas muchas veces se ven transformadas en lugares peligrosos, en los que ocurren robos, homicidios, abusos sexuales, amenazas y daños a bienes materiales, así como formas aún más brutales de violencia (Abramovay, 2005).

En López et al. (2008) se encontró que, ante una situación de mobbing, las estrategias de afrontamiento al problema desde la óptica de la víctima son muy diversas. La estrategia elegida mayoritariamente por las víctimas es no hacer nada (pasiva), hablar con el acosador (activa y asertiva) y ponerlo en conocimiento de la organización o los órganos de representación sindical (denuncia inicial) (López et al., 2008).

En el análisis de los diferentes artículos que se revisaron se encontró que $87.5 \%$ de las personas que presentan algún tipo de mobbing, sean estudiantes, maestros o personal administrativo, optan por no reportarlo a la autoridad universitaria pertinente, ya que se considera un aspecto subjetivo o que es complicado poder demostrarlo de forma objetiva; 
además, también hay instancias dentro de las universidades que no le prestan la importancia que este tipo de violencia necesita.

La mayoría de las personas que padecen mobbing en los espacios universitarios prefieren ignorar al agente o agentes agresores, debido a que no hay cultura de la denuncia y de la defensa de los derechos humanos, a pesar de que la mayoría de las instituciones de educación superior de nuestro país cuenta con dependencias al interior en donde se da seguimiento a este tipo de problemas, con el objetivo de defender la dignidad y los derechos universitarios de las personas afectadas.

Debido a que existen factores psicológicos, subjetivos, simbólicos y éticos puestos en juego en el mundo de las relaciones humanas al interior de la organización, un estudio de este tipo representa una herramienta para llevar a cabo un diagnóstico que dé pauta a un trabajo de intervención organizacional en caso de requerirse (Muñoz y López, 2018).

\section{Conclusiones}

La presencia de mobbing como expresión de violencia física y psicológica en las instituciones de educación superior en México ha presentado un crecimiento exponencial. En la universidad, se manifiesta en todos los campos de relación humana que se pueden presentar: maestro-maestro, maestro-alumno, maestro-personal administrativo, maestropersonal directivo, personal administrativo-personal administrativo, personal administrativopersonal-personal directivo, personal administrativo-alumno, alumno-alumno y alumnopersonal directivo. Al interior de las universidades se deben de establecer políticas que permitan y fomenten las relaciones interpersonales y el mejoramiento del clima educativo y laboral. En el caso de la Universidad Autónoma de Sinaloa, se estableció como una política institucional el Centro de Políticas de Género para la Igualdad entre Mujeres y Hombres. Cada una de las unidades académicas que componen la universidad debe de contar con representantes internos para garantizar la equidad, el respeto y la igualdad entre mujeres y hombres y prevenir cualquier tipo de mobbing que se pueda presentar. 
Revista Iberoamericana

de las Ciencias Sociales y

Humanísticas

ISSN: $2395-7972$

\section{Referencias}

Abramovay, M. (coord. $\left.{ }^{a}\right)$ (2006). Cotidiano das escolas: entre violências. Brasília, Brasil: Unesco / Observatório de Violências nas Escolas / Ministério da Educação/. Recuperado de https://unesdoc.unesco.org/ark:/48223/pf0000145265.

Bowling, N. and Beehr, T. (2006). Workplace harassment from the victim's perspective: A theoretical model and meta-analysis. Journal of Applied Psychology, 91(5), 9981012. Retrieved from https://doi.org/10.1037/0021-9010.91.5.998.

Carrillo, R. (2015). Violencia en las universidades públicas. El caso de la Universidad Autónoma Metropolitana. Ciudad de México, México: Universidad Autónoma Metropolitana. Recuperado de https://www.stunam.org.mx/sa/11carrera/2018/04Violencia_\%20en_\%20las\%20uni versidades_\%20El\%20caso_UAM\%20\%20Rosalia_Carrillo_\%20Meraz.pdf.

Gómez, J. M. (2017). El acoso laboral en la universidad una perspectiva comparada entre Colombia, España y México. Revista Jurídica Derecho, 6(7), 63-81. Recuperado de http://www.scielo.org.bo/scielo.php?script=sci_arttext\&pid=S2413$28102017000200005 \& \operatorname{lng}=$ es\&nrm=iso.

Hutton, B., Catalá, F. y Moher, D. (2016). La extensión de la declaración PRISMA para revisiones sistemáticas que incorporan metaanálisis en red: PRISMA-NMA. Medicina Clínica, 147(6), 262-266. Recuperado de https://doi.org/10.1016/j.medcli.2016.02.025.

Hirigoyen, M. (2001). El acoso moral en el trabajo: Distinguir lo verdadero de lo falso. Barcelona, España: Paidós.

Ibarra, L. M., Escalante, A. E. y Mendizábal, G. (2015). El acoso laboral entre los trabajadores universitarios. Revista Iberoamericana de las Ciencias Sociales y Humanísticas, 4(7). Recuperado de http://riaa.uaem.mx/xmlui/bitstream/handle/20.500.12055/1071/El\%20acoso\%20lab oral $\% 20$ entre $\% 2010$ s\%20trabajadores $\% 20$ universitarios.pdf?sequence $=1 \&$ is Allowe $\mathrm{d}=\mathrm{y}$.

Justicia, F., Benítez, J. L. y Fernández De Haro, E. (2006). Caracterización del acoso psicológico en el contexto universitario. Revista de Psicología del Trabajo y de las Organizaciones, 22(3), 293-308. Recuperado de https://www.redalyc.org/pdf/2313/231317121004.pdf. 
Revista Iberoamericana

de las Ciencias Sociales y Humanísticas

ISSN: $2395-7972$

Leymann, H. (1996). The content and development of mobbing at work. European Journal of Work and Organizational Psychology, 5(2), 165-184. Retrieved from https://www.tandfonline.com/doi/abs/10.1080/13594329608414853.

López, M. Á., Picón, E. y Vázquez, P. (2008). Estudio del acoso psicológico en la universidad pública de Galicia. Revista de Psicología del Trabajo y de las Organizaciones, 24(1), 41-60.

Recuperado

de

http://scielo.isciii.es/scielo.php?script=sci_arttext\&pid=S1576-

59622008000100003.

Montesinos, R. y Carrillo, R. (2011). El crisol de la violencia en las universidades públicas.

El Cotidiano, (170), 49-56. Recuperado de https://www.redalyc.org/pdf/325/32520935006.pdf

Moreno, L., Sepúlveda, L. y Restrepo, L. (2012). Discriminación y Violencia de Género en la Universidad de Caldas. Hacia la Promoción de la Salud, 17(1), 59-76. Recuperado de http://www.scielo.org.co/pdf/hpsal/v17n1/v17n1a05.pdf.

Muñoz, J. P. y López, A. (2018). Mobbing y satisfacción laboral en docentes de Instituciones de Educación Superior del centro de México. Una evidencia empírica sobre su relación. Ciencia y Sociedad, 43(4). Recuperado de https://doi.org/10.22206/cys.2018.v43i4.pp13-21.

Rojas, J., García, B. y Hernández, M. (2019). El Mobbing en trabajadores universitarios: Una revisión sistemática. Propósitos y Representaciones, 7(3). Recuperado de http://revistas.usil.edu.pe/index.php/pyr/article/view/369.

Seijo, C. y Nava, D. (2016). Psicoterror en la Gestión de Educación Superior: Situación Ética desde lo moral. Multiciencias, 16(4). Recuperado de https://www.redalyc.org/jatsRepo/904/90455395009/html/index.html.

Tlalolin, B. F. (2017). ¿Violencia o violencias en la universidad pública? Una aproximación desde una perspectiva sistémica. El Cotidiano, (206), 39-50. Recuperado de https://www.redalyc.org/pdf/325/32553518005.pdf. 
Revista Iberoamericana de las Ciencias Sociales y Humanísticas

\begin{tabular}{|c|c|}
\hline Rol de Contribución & Autor (es) \\
\hline Conceptualización & Brenda García López y Alma Delia Herenas Tapia (Igual) \\
\hline Metodología & Jesús Roberto Garay Núñez \\
\hline Software & $\begin{array}{l}\text { Brenda García López (Principal), Alma Delia Herenas } \\
\text { (Principal) y Jesús Roberto Garay Núñez (Apoyo). }\end{array}$ \\
\hline Validación & $\begin{array}{l}\text { Brenda García López (Principal), Alma Delia Herenas } \\
\text { (Principal) y Jesús Roberto Garay Núñez (Principal). }\end{array}$ \\
\hline Análisis Formal & $\begin{array}{l}\text { Brenda García López (Principal), Alma Delia Herenas } \\
\text { (Principal) y Jesús Roberto Garay Núñez (Principal). }\end{array}$ \\
\hline Investigación & $\begin{array}{l}\text { Brenda García López (Principal), Alma Delia Herenas } \\
\text { (Principal) y Jesús Roberto Garay Núñez (Principal). }\end{array}$ \\
\hline Recursos & $\begin{array}{l}\text { Brenda García López (Principal), Alma Delia Herenas } \\
\text { (Principal) y Jesús Roberto Garay Núñez (Principal). }\end{array}$ \\
\hline Curación de datos & $\begin{array}{l}\text { Brenda García López (Principal), Alma Delia Herenas } \\
\text { (Principal) y Jesús Roberto Garay Núñez (Principal). }\end{array}$ \\
\hline $\begin{array}{l}\text { Escritura - Preparación del } \\
\text { borrador original }\end{array}$ & Brenda García López y Alma Delia Herenas Tapia (Igual) \\
\hline $\begin{array}{l}\text { Escritura - Revisión y } \\
\text { edición }\end{array}$ & Jesús Roberto Garay Núñez \\
\hline Visualización & $\begin{array}{l}\text { Brenda García López (Principal), Alma Delia Herenas } \\
\text { (Principal) y Jesús Roberto Garay Núñez (Principal). }\end{array}$ \\
\hline Supervisión & Jesús Roberto Garay Núñez \\
\hline $\begin{array}{l}\text { Administración de } \\
\text { Proyectos }\end{array}$ & Jesús Roberto Garay Núñez \\
\hline Adquisición de fondos & $\begin{array}{l}\text { Brenda García López (Principal), Alma Delia Herenas } \\
\text { (Principal) y Jesús Roberto Garay Núñez (Principal). }\end{array}$ \\
\hline
\end{tabular}

